\title{
Using a Deep Learning Algorithm to Improve the Results Obtained in the Recognition of Vessels Size and Trajectory Patterns in Shallow Areas Based on Magnetic Field Measurements Using Fluxgate Sensors
}

\author{
Marina Pérez ${ }^{(\mathbb{1}}$, Juan Parras, Santiago Zazo, Member, IEEE , \\ Iván A. Pérez Álvarez, Member, IEEE, and María del Mar Sanz Lluch
}

\begin{abstract}
Safety in coastal areas such as beaches, ports, pontoons, etc., is a current problem with a difficult solution and on which many organizations are putting efforts in terms of technological innovation. In this work the design of a possible solution based on magnetic sensors is presented. First, a study has been made of the type of sensors that best suit the application based on parameters such as sensitivity, the allowed bandwidth of excitation, price or physical construction. Then the system of excitation of the sensors and signal measurement is presented. To justify the design, a series of simulations of magnetic field variations have been carried out in the presence of large objects of conductive material, in the vicinity of the measuring points. With these data a mathematical model has been established that allows the identification of the dimensions and position of the object through triangulation and knowing only the data of the magnetic field. It was found that although this method seems quite effective, it has a significant error, so another method based on neural networks was developed also using data from the simulations. This method seems to yield much better and more reliable results.
\end{abstract}

Index Terms-Magnetic sensors, fluxgate, magnetic simulations, vessels, deep learning, deep neural networks, pattern recognition.

\section{INTRODUCTION}

$\mathbf{T}$ HE need to control vessels in shallow maritime areas such as coasts, ports or pontoons, begins to acquire a great

Manuscript received February 7, 2019; revised October 11, 2019 and June 18, 2020; accepted October 30, 2020. This work was supported in part by Programa Estatal de Investigación, Desarrollo e Innovación Orientada a los Retos de la Sociedad and in part by the Plan Estatal de Investigación Científica y Técnica y de Innovación 2013-2016 under Grant TEC2016-76038-C3-2-R. The Associate Editor for this article was E. Kaisar. (Corresponding author: Marina Pérez.)

Marina Pérez and María del Mar Sanz Lluch are with the School of Telecommunication Engineering, Universidad Politécnica Development Madrid, 28040 Madrid, Spain (e-mail: marina.perez@upm.es; mar.sanz.lluch@upm.es).

Juan Parras and Santiago Zazo are with the Information Processing and Telecommunications Center (IPTC), Universidad Politécnica de Madrid, 28040 Madrid, Spain (e-mail: j.parras@upm.es; santiago@gaps.ssr.upm.es).

Iván A. Pérez Álvarez is with the Institute for Technological Development and Innovation in Communications (IDeTIC), Universida Politécnica de Las Palmas de Gran Canaria, 35001 Las Palmas de Gran Canaria, Spain (e-mail: ivan.perez@ulpgc.es).

Digital Object Identifier 10.1109/TITS.2020.3036906 interest. Because of that, within the HERAKLES [1] project, we have worked on the sensorization of a network of nodes to achieve a method that allows for the surveillance, identification and monitoring of objectives. The information obtained in each of the nodes is shared and used collaboratively in such a way that it is mandatory to speak of a network structure.

Currently there is a great development in the field of underwater networks based on various types of sensors [2], [3] for networks of detection, identification, surveillance or aid to navigation [4]-[6] or for other applications such as scientific use [7] or as communication networks for various uses [8].

Of all the different types of communications nodes used in underwater applications, the most widespread for detection and identification are acoustic based sensors, due to the high propagation speed of sound in water $(1500 \mathrm{~m} / \mathrm{s})$. It is a relatively old technology that has been perfected for many years. Nowadays, new modem models are appearing, and are contributing to improve the acoustic sensors based networks [9] while the transducers are usually called hydrophones. This type of sensors is made using (in most of the cases) piezoelectric materials to convert mechanical energy in electrical energy. It allows to register information that comes as a sound wave (pressure in water particles). There are lots of examples of applications which use this kind of sensors as main elements in underwater acoustic networking. [10].

Therefore, the most used Wireless Sensor Network (WSN) for the underwater medium is the Acoustic Underwater WSN (AUWSN) due to the lower attenuation of acoustic waves in the sea water. Hence, it is no surprise that AUWSN are used for many tasks, such as monitoring applications, disaster management, military related applications, assisted navigation, localization and tracking [1]. There are several localization methods used in AUWSN [2]-[5], which can be broadly classified into time or angle of arrival methods, and received power. In time of arrival localization methods it is frequent to use anchor nodes [6], although self-localization algorithms exists to alleviate the deployment cost of such anchor nodes [7]. The methods based on time or angle of arrival are more popular due to the time-varying path loss 
and the strong multipath effect of the underwater channel, as [3] shows; however, the powerful approximation capabilities of deep neural networks are being used to locate using the received acoustic power, as [8]-[10] show.

Even though sonar and hydrophones are the preferred sensors for most developments in underwater acoustic networks, networking that uses this type of sensors has problems as path loss, noise, multipath, propagation delay and other problems related with Doppler effect [22].

Currently the acoustic sonar technology fully justifies its use as a widely used element within the networks of identification, detection of objects and many of other applications [11], [12], but there are other alternatives.

In this work we are going to present two important novelties: on the one hand, instead of acoustic, we have developed a network whose propagation is based instead on electromagnetic fields. On the other hand, we propose the installation of a series of magnetic sensors combined with hydrophones, in the nodes of a submerged network, in such a way that the information obtained from both sensors allows to improve the detection and identification that was done until now using only sonar technology.

The advantages of EM network have been properly addressed in [26] as the core of HERAKLES [1] project, where a theoretical and simulated propagation model was cross-checked with extensive measurement campaigns showing interesting properties about coverage, antenna design and link characteristic.

The election of magnetic sensors to develop the network has been carried out considering the resolution that they allow. The measure that sensors provide is defined in terms of magnetic field variations allowing measures of units of pT. Even slightly worse resolutions permit estimate the shape of the object, its size or even its magnetic structure [23], which is an information difficult to obtain using acoustic technology. In addition, magnetic field variation reaches the sensor as a nonmechanical wave consequently in terms of the channel through wave propagates, there is no possibility of dispersion because certain effects like water composition variations, existence of unwanted elements into the communication channel, etc. This is why this paper justifies the use of magnetic sensors as unique devices to get the detection and complete characterization of vessels. Information that, in some cases, could be complemented with information obtained using acoustic resources.

The magnetic sensors will offer information regarding the size and distance of the object [23] and by processing the received signal it will be possible to find out the path that is being followed within the area of the network. In this way it can be obtained information related to the navigation of boats (trajectory, speed, etc.) whose size makes identification difficult through other methods, besides being considered noncollaborative vehicles, that is, they do not have any device installed that contains and transmits information accessible by the control authorities such as ports or other surveillance entities.

This work has been divided into 3 parts. In the first part we talk about hardware necessary to handle the magnetic sensors: a small explanation about the sensors used, the signal excitation and magnetic field measurement electronics and the mention of a calibration circuit necessary to cancel the possible perturbations to the extent caused by the existence of objects in the measurement environment that are not vessels. The second part is the simulations that have been done to justify the use of the magnetic sensors together with the analysis of them and the description of the triangulation process that should be done with the sensed nodes to obtain the size of the object and its location within of the network.

As an alternative to this procedure we have developed another method using the data obtained in the simulations as inputs in a neural network that results in more precise and extensive information about the size of the vessel, its location and the trajectory that follows. The third part of this work describes the use of the neural network and justifies its use as a universal function approach.

\section{SENSORS COMPARATION}

There are many types of magnetic sensors. Most of them are commercial and are sold with integrated electronic. Others are manufactured more specifically for each application, adapting them to requirements of size, weight, sensitivity, etc.

In the work presented in [24] a compilation of the main characteristics of different types of magnetic sensors is made. For the required application, the use of magnetoresistive anisotropy (AMR) sensors and fluxgate sensors was evaluated. The benefits that each one allowed in the following aspects were studied:

1.- Sensitivity and resolution required. Detection occurs when measuring variations from $10 \mathrm{nT}$ to $100 \mathrm{nT}$ over the Earth's magnetic field estimated at $64 \mu \mathrm{T}$.

2.- Working bandwidth. The sensors are placed at a certain distance from the control unit, so the excitation of them will be in AC to avoid noise.

3.- Physical characteristics. The space provided by the communications node limits the dimensions of the sensors in size and weight.

4.- Price of the sensors.

The size and weight characteristics of the sensors, as well as their price, do not seem to be decisive in ruling out one type or the other. The fluxgate sensors are so much more expensive the more sensitivity they require, however, the associated electronics are simpler than the AMRs, which are manufactured as integrated devices but require a more complex setup. In terms of size, the fluxgate are as big as the ferrite cores that make them up. In addition, aspects like the type of core, number of turns by winding in the primary and secondary influence on performance that are decisive as sensitivity or resolution. But, even in very specific requirements, the dimensions of the fluxgate do not make it impossible to implement.

The sensors will be placed at a distance that will vary between 6 and 30 meters with variations due to the oscillation of the boat due to the waves, estimated at 1 meter. At this distance, the field induced by the object measuring the sensor is between 100 and 200nT for a standard object size, and the variation per meter is estimated between 10 and 50nT, so the resolution and sensitivity parameters are determinant to choose 
a type of sensor. The fluxgate allows resolutions lower than $100 \mathrm{pT}$ while the AMR have resolutions of 8 ' $5 \mathrm{nT}$. In the case of sensitivity, the first can give $1 \cdot 10^{5} \mathrm{~V} / \mathrm{T}$ while the AMR will give $50 \mathrm{~V} / \mathrm{T}$.

Finally, the bandwidth is compared. This parameter is important because it gives the frequency values in which it is preferable to make the sensor work. In order to avoid interferences with communications between nodes, an operating band is chosen around the tens of $\mathrm{KHz}$. It has been thought of a fundamental tone frequency of around $15 \mathrm{KHz}$. This frequency does not interfere with any other frequency used in the rest of the system and is suitable for excitation of the magnetic core. The bandwidth of the fluxgate extends from $5 \mathrm{KHz}$ and the bandwidth of the AMR goes from direct current to $5 \mathrm{MHz}$ (approximately).

Therefore, fluxgate sensors are chosen because they offer the best performance in terms of resolution and sensitivity, with the rest of the parameters being equally valid in the two types of sensors.

\section{Proposed Solution}

\section{A. Sensor Head}

The sensor used is a fluxgate formed by two cores of magnetic material placed against each other, to eliminate the presence of the fundamental tone, and a secondary winding. Also included is a ground field compensation system (zero calibration) composed of a third coil. The model is similar to the one used in [23] for the control of air traffic in airports.

To improve the sensitivity, between 7 and $101.5 \mathrm{~mm} \times$ $35 \mathrm{~mm}$ tapes of METGLAS 2714A, which is an alloy of amorphous material based on cobalt, was used as a core of magnetic material. For the windings of the sensor itself as well as the compensation coils, $0.02 \mathrm{~mm}$ wire was used and an average of 50 turns were given per core of the primary and around 20 turns for each calibration coil. For the secondary winding the number of turns was estimated at 30 .

\section{B. Electronics}

The second harmonic control and measurement electronics that has been designed are divided into two blocks. On the one hand, the elements responsible for the generation of the excitation signal of the sensor and on the other hand the devices responsible for the detection, measurement and storage of the voltage in the secondary. The design is based on a lockin amplifier implemented with a full-wave rectifier as will be explained below. The general scheme can be seen in figure 1 .

The element that controls the circuit is a microcontroller configured in slave mode with a serial connection of type SPI through which the signal generation devices and the reading $\mathrm{ADC}$ of the second harmonic are controlled.

Loading information in the registers of the two oscillators, two signals are generated. One will be the excitation of the sensor and the other will be the reference for the lock-in. The frequency, phase and shape of the signal are controlled in the oscillator of the sensor excitation signal. The amplitude of the signal is controlled by loading the DAC registers. In the last step prior to entering the sensor head, the signal is

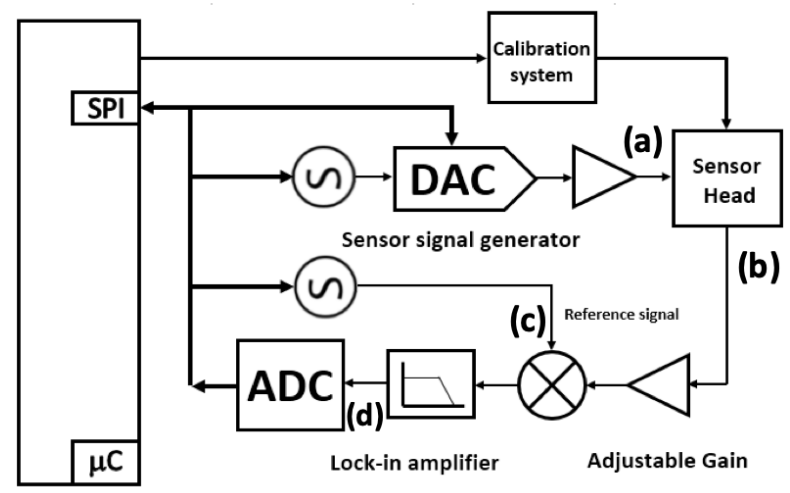

Fig. 1. System block diagram.

amplified by connecting a power amplifier. Both the generator of the magnetic sensor signal and the generator of the reference signal are connected to a $1 \mathrm{MHz}$ clock that synchronizes them.

The sensor output (secondary) is connected to a variable gain amplifier and the output to a full wave rectifier implemented by means of a demodulator and an active low pass filter with cutoff frequency below $5 \mathrm{KHz}$. This filter is formed by a filter of $2^{\text {nd }}$ order and another of $1^{\text {st }}$ order.

The last element of the chain is formed by a 12-bit digital analog converter that sends the conversion data to the microcontroller through the SPI connection.

A calibration of field compensation system is also connected, and it is composed of a coil controlled by a comparison system implemented in the micro-sensor itself and a digitalanalog converter that controls a current regulation system by means of which it can be connected to the sensor head. It can compensate the level of voltage that appears on the secondary sensor and that is a consequence of Earth's magnetic field and other elements of the sensor's environment.

\section{Operation Mode}

The system works by means of a signal loop. That is, an excitation signal is generated that enters the primary of the sensor. After this phase the calibration is carried out to make sure that there is no voltage in the secondary that can vary the actual magnetic field measurement value. Once the calibration process is finished, object detection is started using the algorithm shown in Figure 2.

Sensors are constantly measuring. To do that a loop like showed in Figure 2 is executed. The process to obtain a measure starts with a change in the phase of the reference signal which is compared to the signal received at the magnetic sensor. Factors related to the physical characteristics of the channel through which magnetic field wave travels, affect only the phase of the signal received and its amplitude. We know the attenuation coefficient of the channel [26] and because the measurement system is based on a lock-in amplifier (Figure 1), we can eliminate the variations in the phase of the signal due to wave propagation. Then in each loop execution, the information obtained by the sensors is adjusted applying the attenuation coefficient and calculating the real phase of the signal, varying the phase of the signal reference, until the voltage given by the measurement system will be maximum. 


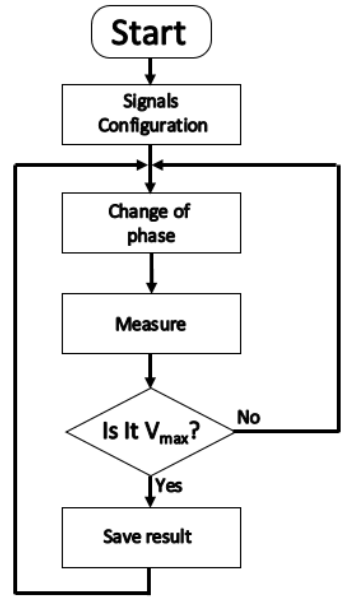

Fig. 2. Algorithm implemented in the microcontroller for the detection and measurement of the second harmonic.

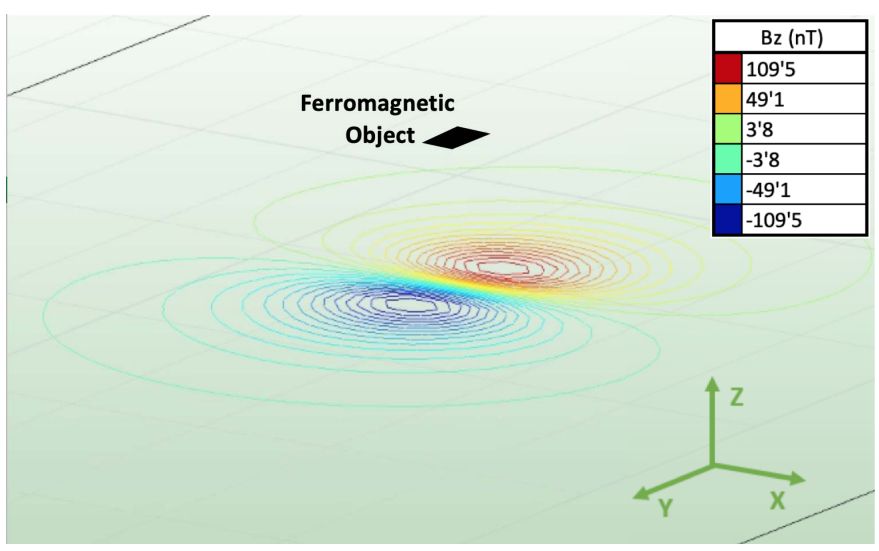

Fig. 3. Example of data representation using AMPERES.

Because of that the voltage given by the measure system is only related to the variation of magnetic field generated by the object we want to detect and characterize.

\section{Simulations}

A series of simulations have been carried out to obtain data about the magnetic field that would allow an algorithm to be created to determine the size of the vessel and its approximate trajectory.

The simulations have been done with AMPERES [24] software. In this program Maxwell's equations for the magnetic field are solved by meshing the surfaces that are to be studied. Four iron-shaped ferromagnetic objects of dimensions $4 \mathrm{~m} \times$ $2 \mathrm{~m}, 8 \mathrm{~m} \times 4 \mathrm{~m}, 20 \mathrm{~m} \times 10 \mathrm{~m}$ and $40 \mathrm{~m} \times 20 \mathrm{~m}$ have been simulated (represented in figure 3 as the blak square). These dimensions have been chosen because they are considered the most representative of the actual boat sizes. It has been simulated at different depths of the seabed: $7,5 \mathrm{~m}, 10 \mathrm{~m}, 20 \mathrm{~m}$, and $30 \mathrm{~m}$, as samples that allow to interpolate all the rest of distances.

The obtained results are matrices of around 60000 rows with 4 columns where the 3 spatial coordinates (represented in meters) and the data of magnetic field in that point, are represented. In figure 3 we show an example of how simulations look like. This figure is a representation of the value of magnetic field $\mathrm{B}$ in each point which coordinates are registered in the matrix mentioned before.

Once the results were obtained, they were processed using two different methods. On the one hand, there is a mathematical analysis based on the equation that governs the behavior of a magnetic field in the presence of an object and the and on the other hand, a second method uses a neural network to develop an algorithm that directly uses the data obtained from the simulations.

\section{A. Mathematical Analysis}

This method of analysis is based on the equation that defines the influence of an object on the magnetic field at a point.

The value of the $B$ field in a position $r$ (which coordinates are: $x, y$ and $z$ ), is obtained by calculating the rotational of the magnetic potential vector $(A)$ that is defined as shown in equation 1.1 .

$A(x, y, z)=\frac{\mu_{0}}{4 \pi} \int_{V_{0}} \frac{\nabla^{\prime} \times M}{\left|r-r^{\prime}\right|} d v^{\prime}+\frac{\mu_{0}}{4 \pi} \int_{S_{0}} \frac{M \times n}{\left|r-r^{\prime}\right|} d a^{\prime}$

$V_{0}$ is the total volume of studied body and $S_{0}$ is its surface. This equations shows that $A$ only depends on volume of body and its surface.

Once obtained $A$ and doing the necessaries calculations, the rotational is calculated as can be seen in equation 1.2.

$$
B(r)=\nabla \times A=\frac{\mu_{0}}{4 \pi} \int_{V_{0}} \nabla \times\left[M \times \frac{\left(r-r^{\prime}\right)}{\left|r-r^{\prime}\right|^{3}}\right] d v^{\prime}
$$

Applying the properties of the divergence, 1.2 can be written as two terms.

$$
B(r)=B_{I}(r)+B_{I I}(r)
$$

The first term is described in equation 1.4.

$$
B_{I}(r)=\frac{\mu_{0}}{4 \pi} \int_{V_{0}} M\left(r^{\prime}\right) 4 \pi \delta\left(r-r^{\prime}\right) d v^{\prime}
$$

The calculation of this integral will result in the total field that is seen from the position $r$ due to the vector $M$ in the position $r^{\prime}$ (object position), or, in other words, the total magnetization of the body whose effect in the magnetic field is to be studied:

$$
B_{I}(r)=\mu_{0} M(r)
$$

The second term can be written as seen in equation 1.6.

$$
B_{I I}(r)=-\mu_{0} \nabla \frac{1}{4 \pi} \int_{V_{0}} M\left(r^{\prime}\right) \cdot \frac{\left(r-r^{\prime}\right)}{\left|r-r^{\prime}\right|^{3}} d v^{\prime}
$$

Knowing that $\varphi^{*}$ is a scalar field that solves the magnetic potential due to the magnetic material, as can be seen in equation 1.7 .

$$
\varphi^{*}(r)=\frac{1}{4 \pi} \int_{V_{0}} M\left(r^{\prime}\right) \cdot \frac{\left(r-r^{\prime}\right)}{\left|r-r^{\prime}\right|^{3}} d v^{\prime}
$$

1.7 can be rewritten as in 1.8 .

$$
\varphi^{*}(r)=\frac{1}{4 \pi} \int_{S_{0}} \frac{M \cdot n}{\left|r-r^{\prime}\right|} d a^{\prime}-\frac{1}{4 \pi} \int_{V_{0}} \frac{\nabla^{\prime} \cdot M}{\left|r-r^{\prime}\right|} d v^{\prime}
$$




\begin{tabular}{|r|r|}
\hline distance $(\mathrm{m})$ & \multicolumn{1}{|l|}{$\Delta \mathrm{B}(\mathrm{nT})$} \\
\hline 7,5 & 277,1 \\
\hline 10 & 156,1 \\
\hline 20 & 32,32 \\
\hline 30 & 11,29 \\
\hline
\end{tabular}

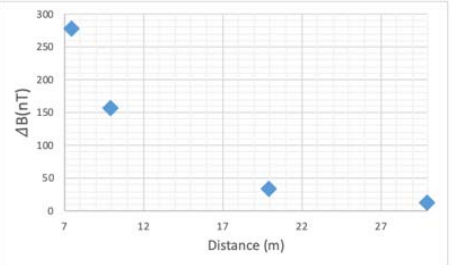

Fig. 4. Example of table where it is represented the maximum field variation for each distance, for a concrete object size.

And the values:

$$
\rho_{M}\left(r^{\prime}\right) \equiv-\nabla^{\prime} M\left(r^{\prime}\right) ; \quad \sigma_{M}\left(r^{\prime}\right) \equiv M\left(r^{\prime}\right) \cdot n
$$

Are defined as the magnetic pole density $\rho_{M}$ and the surface density of the magnetic pole intensity $\sigma_{M}$ respectively.

The total magnetic field at a point $r$ defined by its coordinates $x, y$ and $z$ as can be seen in 1.9.

$$
\begin{aligned}
B(r)= & \frac{\mu_{0}}{4 \pi} \int_{V_{0}} \rho_{M} \frac{\left(r-r^{\prime}\right)}{\left|r-r^{\prime}\right|^{3}} d v^{\prime} \\
& +\frac{\mu_{0}}{4 \pi} \int_{S_{0}} \sigma_{M} \frac{\left(r-r^{\prime}\right)}{\left|r-r^{\prime}\right|^{3}} d a^{\prime}+\mu_{0} M(r)
\end{aligned}
$$

$M$ is a function defined around $\mathrm{x}, \mathrm{y}$ and $\mathrm{z}$ and that will define the way in which the magnetization is distributed in a material.

The fact that $M$ is a function involves talking about a magnetic pole density and a surface density, such as the divergence or evolution of the function in each of the axes, and the effective value of that function on the surface of the material.

In 1.9, the dependence of $B$ with distance can also be observed. The magnetic field decreases with distance in a cubic factor. At close range the first two terms acquire relevance. This causes that when the object is near to the point where the field is being measured, it is possible to differentiate better between the effect produced by the surface distribution and the volumetric distribution.

This dependence on $B$ is also interpreted as an effect produced by an object whose size can be compared with distance. Then the analysis must be done from the perspective of the size of the body comparing it with the distance to the point of measurement.

\section{Problem Solution And Analysis of the Results}

\section{Non-linear least squares approximation}

The variation behavior of magnetic field $B$ due to the presence of an object place closed to the measuring point, is represented by the maximum of this variation obtained in each of the simulations. In this way we can establish a total of 4 tables with values (for each simulated object size) formed by 4 pairs of points: distance $r$, maximum field variation $\Delta B$ [figure 4].

The data for the 4 tables taken from the 4 simulated object sizes, is not enough to do an in-depth study of the magnetic field behavior and its dependence on the size of the object, which causes the field variations. In order to get additional information about this dependency, we have to interpolate the
TABLE I

Parameter Values of Vector $\beta$ For Each Object Size

\begin{tabular}{|l|l|l|l|}
\hline Size $\left(\mathrm{m}^{2}\right)$ & a & b & c \\
\hline 2 & $5^{\prime} 3 \cdot 10^{4}$ & $-2^{\prime} 83$ & $-5^{\prime} 09$ \\
\hline 8 & $7^{\prime} 11 \cdot 10^{4}$ & $-2^{\prime} 8$ & $-1^{\prime} 11$ \\
\hline 32 & $2^{\prime} 17 \cdot 10^{5}$ & $-2^{\prime} 65$ & $-99^{\prime} 15$ \\
\hline 200 & $1^{\prime} 39 \cdot 10^{4}$ & $-1^{\prime} 92$ & $-99^{\prime} 65$ \\
\hline
\end{tabular}

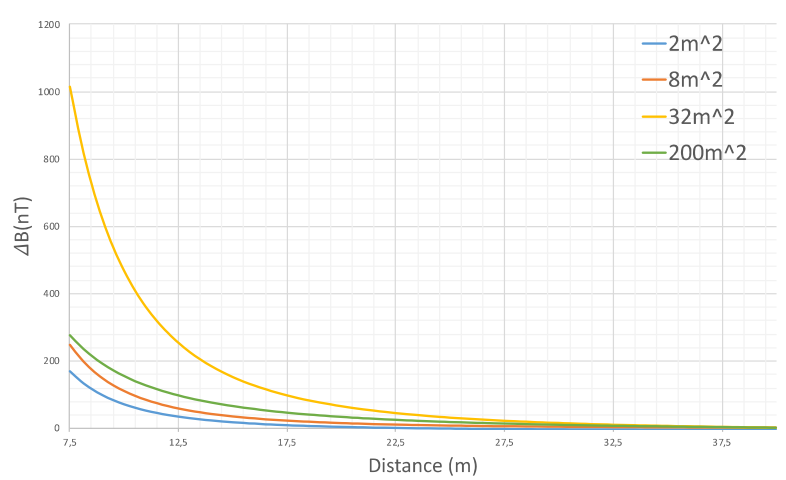

Fig. 5. Representation of magnetic field $B$ and distance $r$ between object and measuring point for every object size simulated.

information from these pairs of values $(r, \Delta B)$ by analyzing the trend of each variation.

The procedure we used was interpolate using a surrogate function like a polynomial, using non-linear least squares approximation, which minimizes the error margin of each sample. The approximation with minimal error is $2^{\text {nd }}$ degree power function. This type of function has the mathematical definition showed in equation 1.10.

$$
\Delta B(r, a, b, c)=a r^{b}+c
$$

where vector $\beta$ is formed by parameters $a, b$ and $c$, and $r$ is the distance. Table I shows the parameter values for each simulated object size.

Identification of vessels and their position in the network of nodes.

Taking the value of $a, b$ and $c$ parameters, we calculated the variation of magnetic field B for each distance $r$ and object size $m$. See equation 1.11 .

$$
\Delta B=f_{\theta}(r, m)
$$

where $\theta$ represents the 2 nd degree power function used to approximate the trend of the variation of magnetic field $B$.

To simplify the calculations we will only use distances between 7'5 $\mathrm{m}$ (minimum distance in simulations) and $40 \mathrm{~m}$ (maximum distance taking into account the distance between nodes).

Figure 5 shows that there is no an univocal relation between the group of image values $\Delta B$ and the group of source values $(r, m)$.

To solve this problem we will use a method based on the correlated information given by a group of sensors.

When the sensors are deployed on the seabed and are included within the network, they execute an algorithm that allows them to know the distance they are from the nodes next to them [18]. These distances are established between 
20 and 25 meters maximum. In groups of three sensors one of them is identified as the origin of coordinates and together with the other two form a triangle. Because we are using an EM communication system [26], once deployed each node, the procedure to identify the vessel (size) and give an approximately value of position where it is located, starts.

If we have a measure of the variation of magnetic field $B$ $\left(\Delta B_{i}\right)$ for a determined object size $m^{*}$ obtained by sensor $i$, equation 1.11 can be particularized to:

$$
\Delta B_{i}=f_{\theta}\left(r_{i}, m^{*}\right)
$$

We can observed that equation 1.12 represents a curve as the intersection between $f_{\theta}\left(r_{i}, m^{*}\right)$ and a horizontal plane that has a value equal to $\Delta B_{i}$. Given $\Delta B_{i}$ and a know value of $m^{*}$, and using equation 1.13 we can calculate the distance between the object and sensor $i$.

$$
r_{m}=f_{\theta}^{-1}\left(\Delta B_{i}, m^{*}\right)
$$

However, as in practice we do not know $m^{*}$ there could be a set of candidates $\left(r_{m}, m\right)$ the fulfills (equation 1.13).

On the other hand, if we have a set of sensors, the localization problem is defined by a set of equations. (Equation 1.14).

$$
\Delta B_{i}=f_{\theta}\left(r_{i}, m^{*}\right) \quad \forall i \epsilon(1, M)
$$

Instead of using this notation (equation 1.14) we will identify the position of each sensor with coordinates $z_{i}=\left(x_{i}, y_{i}\right)$, and thus $\rho(x, y)$ will be the geometric place that meets the mathematical condition (equation 1.15):

$$
\left\|z_{i}-\rho\right\|^{1}=\left(x_{i}-x\right)^{2}+\left(y_{i}-y\right)^{2}=r_{i}
$$

Which is the equation describing a circumference. However, this equation has implicitly the unknown $m^{*}$, due to the fact that it couldn't be directly applied. To understand more clearly the optimization process we will suppose that there are 3 nodes. Using this architecture, the equations to verify are (equation 1.16):

$$
\left\|z_{i}-\rho\right\|^{1}=\left(x_{i}-x\right)^{2}+\left(y_{i}-y\right)^{2}=\left(f_{\theta}^{-1}\left(\Delta B_{i}, m^{*}\right)\right)^{2}
$$

If we solve a set of systems formed by the pairing of previous equations (equations 1.17):

Sensors 1 and 2:

$$
\begin{aligned}
& \left\{\begin{array}{l}
\left(x_{1}-x\right)^{2}+\left(y_{1}-y\right)^{2}=\left(f_{\theta}^{-1}\left(\Delta B_{1}, m^{*}\right)\right)^{2} \\
\left(x_{2}-x\right)^{2}+\left(y_{2}-y\right)^{2}=\left(f_{\theta}^{-1}\left(\Delta B_{2}, m^{*}\right)\right)^{2}
\end{array}\right. \\
& \rightarrow 2 \text { solutions }\left\{\begin{array}{l}
\alpha_{12}=\left(x_{12}, y_{12}\right) \\
\rho^{*}=\left(x^{*}, y^{*}\right)
\end{array}\right.
\end{aligned}
$$

Sensors 1 and 3:

$$
\begin{aligned}
& \left\{\begin{array}{l}
\left(x_{1}-x\right)^{2}+\left(y_{1}-y\right)^{2}=\left(f_{\theta}^{-1}\left(\Delta B_{1}, m^{*}\right)\right)^{2} \\
\left(x_{3}-x\right)^{2}+\left(y_{3}-y\right)^{2}=\left(f_{\theta}^{-1}\left(\Delta B_{3}, m^{*}\right)\right)^{2}
\end{array}\right. \\
& \rightarrow 2 \text { solutions }\left\{\begin{array}{l}
\alpha_{13}=\left(x_{13}, y_{13}\right) \\
\rho^{*}=\left(x^{*}, y^{*}\right)
\end{array}\right.
\end{aligned}
$$

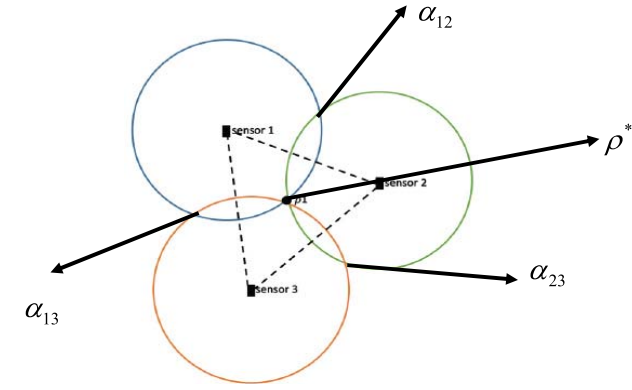

Fig. 6. Resolution of optimization process. The process has finished and a result of $m^{*}$ and $r_{i}$ is achieved because we have obtained $\rho^{*}$.

Sensors 2 and 3 :

$$
\begin{aligned}
& \left\{\begin{array}{l}
\left(x_{2}-x\right)^{2}+\left(y_{2}-y\right)^{2}=\left(f_{\theta}^{-1}\left(\Delta B_{2}, m^{*}\right)\right)^{2} \\
\left(x_{3}-x\right)^{2}+\left(y_{3}-y\right)^{2}=\left(f_{\theta}^{-1}\left(\Delta B_{3}, m^{*}\right)\right)^{2}
\end{array}\right. \\
& \rightarrow 2 \text { solutions }\left\{\begin{array}{l}
\alpha_{23}=\left(x_{23}, y_{23}\right) \\
\rho^{*}=\left(x^{*}, y^{*}\right)
\end{array}\right.
\end{aligned}
$$

Notice that for each system we can obtain two solutions. One of these is inside the triangle connecting the positions of the sensors, and, the other solution, outside this triangle. Under the hypothesis that the $\Delta B$ measurements are correct and the value of $m^{*}$ is known, the solutions inside the triangle have to converge at a single point and this is, then the solution to the problem. It is showed in figure 6.

However in practice, we can not follow this approach because, precisely the value of $m^{*}$ is unknown. If we select a random value of $m$ and we repeat the process we will obtain (equations 1.18):

Sensors 1 and 2:

$$
\begin{aligned}
& \left\{\begin{array}{l}
\left(x_{1}-x\right)^{2}+\left(y_{1}-y\right)^{2}=\left(f_{\theta}^{-1}\left(\Delta B_{1}, m^{*}\right)\right)^{2} \\
\left(x_{2}-x\right)^{2}+\left(y_{2}-y\right)^{2}=\left(f_{\theta}^{-1}\left(\Delta B_{2}, m^{*}\right)\right)^{2}
\end{array}\right. \\
& \rightarrow 2 \text { solutions }\left\{\begin{array}{l}
\alpha_{12}=\left(x_{12}, y_{12}\right) \\
\beta_{12}=\left(x_{12}^{\prime}, y_{12}^{\prime}\right)
\end{array}\right.
\end{aligned}
$$

Sensors 1 and 3:

$$
\begin{aligned}
& \left\{\begin{array}{l}
\left(x_{1}-x\right)^{2}+\left(y_{1}-y\right)^{2}=\left(f_{\theta}^{-1}\left(\Delta B_{1}, m^{*}\right)\right)^{2} \\
\left(x_{3}-x\right)^{2}+\left(y_{3}-y\right)^{2}=\left(f_{\theta}^{-1}\left(\Delta B_{3}, m^{*}\right)\right)^{2}
\end{array}\right. \\
& \rightarrow 2 \text { solutions }\left\{\begin{array}{l}
\alpha_{13}=\left(x_{13}, y_{13}\right) \\
\beta_{13}=\left(x_{13}^{\prime}, y_{13}^{\prime}\right)
\end{array}\right.
\end{aligned}
$$

Sensors 2 and 3:

$$
\begin{aligned}
& \left\{\begin{array}{l}
\left(x_{2}-x\right)^{2}+\left(y_{2}-y\right)^{2}=\left(f_{\theta}^{-1}\left(\Delta B_{2}, m^{*}\right)\right)^{2} \\
\left(x_{3}-x\right)^{2}+\left(y_{3}-y\right)^{2}=\left(f_{\theta}^{-1}\left(\Delta B_{3}, m^{*}\right)\right)^{2}
\end{array}\right. \\
& \rightarrow 2 \text { solutions }\left\{\begin{array}{l}
\alpha_{23}=\left(x_{23}, y_{23}\right) \\
\beta_{23}=\left(x_{23}^{\prime}, y_{23}^{\prime}\right)
\end{array}\right.
\end{aligned}
$$

Figure 7 represents this other situation:

We are still referring to solutions outside the triangle using the same notation as in first situation. We observed quite 


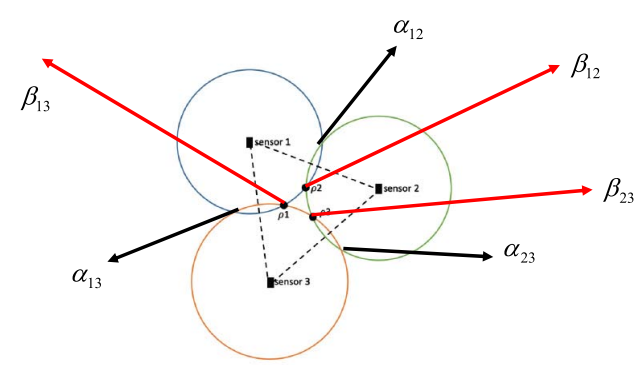

Fig. 7. Representation of solutions obtained with equations 1.18.

a difference situation in terms of solutions inside the triangle, because each system of equations gives different points (named using $\beta_{i j}$ )

If the selected value of $m$ is quite different from $m^{*}, \beta_{i j}$ points will be far from each other. But if $m \approx m^{*}$, the distance between these points decreases. Because of this an algorithm to find the right solution can be proposed. We must consider certain details:

1- At the moment of starting the algorithm we must find the smallest value of $m_{0}$ (within the range of accuracy of the measurement systems) to achieve two solutions $\left(\alpha_{i j}, \beta_{i j}\right)$ for each system of equations.

2- It is not possible to apply a gradient approach technic because we do not have the analytic expressions: $r_{m}=$ $f_{\theta}^{-1}\left(\Delta \mathrm{B}_{\mathrm{i}}, \mathrm{m}\right)$ consecuently we are going to use a grid of points: $m_{k}=m_{0}+k \Delta m$ selecting $\Delta m$ properly.

3- Cost function will minimize the distances between points $\beta_{i j}$ :

$$
J(m)=\sum_{i=1}^{N-1} \sum_{j=i+1}^{N}\left\|\beta_{i j}\right\|^{2}
$$

This is a convex function, having the minimum value at $J\left(m^{*}\right) \leq J(m) \forall m>0$.

4.- The final result will be obtained when the increment of cost function between consecutive interactions keeps below the threshold value $\varepsilon$.

\section{Algorithm explanation and obtained results.}

Algorithm is described as follows:

Initialize $m_{0}, \Delta m, \varepsilon$

$m=m_{0}, J_{\text {ref }}=0$

While not finish

$m=m+\Delta m$

Find: $\left\{\begin{array}{l}\left(x_{i}-x\right)^{2}+\left(y_{i}-y\right)^{2}=\left(f_{\theta}^{-1}\left(\Delta B_{i}, m^{*}\right)\right)^{2} \\ \left(x_{j}-x\right)^{2}+\left(y_{j}-y\right)^{2}=\left(f_{\theta}^{-1}\left(\Delta B_{j}, m^{*}\right)\right)^{2}\end{array}\right.$ $\rightarrow \beta_{i j} i=0, \ldots, N-1 . j=i+1, \ldots, N$

Calculate $J(m)=\sum_{i=1}^{N-1} \sum_{j=i+1}^{N}\left\|\beta_{i j}\right\|^{2}$

if $\left|J_{\text {ref }}-J(m)\right|<\varepsilon$ then $m^{*}=m$; break while

else $J_{\text {ref }}=J(m)$

end if

end while

After initializing values $m_{0}, \Delta m$ and $\varepsilon$, the algorithm establishes the loop start with $m=m_{0}$ and $J_{r e f}=0$. In each loop execution the value of $m$ is increased in $\Delta m$. After

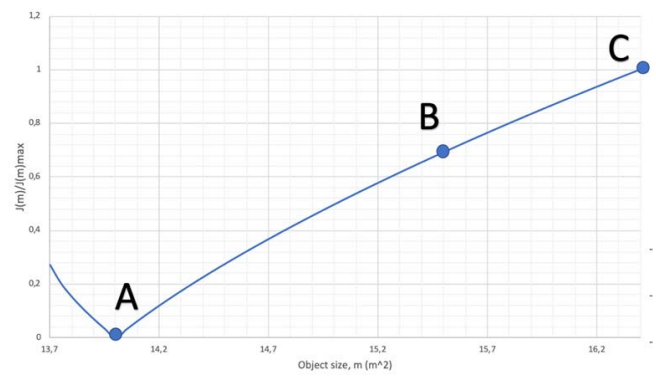

Fig. 8. Nomalizated results of $J(m)$ for an object size of $14 \mathrm{~m}^{2}$.

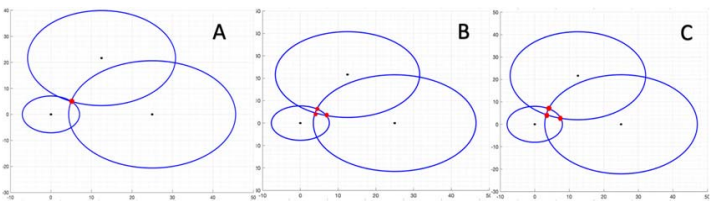

Fig. 9. Representation of the convergence o $\beta_{i j}$ solutions for an object size of $14 \mathrm{~m}^{2}$.

initialization three systems of equation are solved (one for each pair of sensors) and two solutions are obtained: $\alpha_{i j}$ and $\beta_{i j}$ that are the solution outside and inside the triangle formed joining sensor positions, respectively. The next step is to solve the cost function using interior points. The magnitude obtained $J(\mathrm{~m})$ can be compared with reference magnitude $J_{\text {ref }}$ and, if the cost function solution $J(m)$ is below threshold $\varepsilon$, the final solution is $m^{*}$ and the algorithm is over, if not, reference magnitude $J_{r e f}$ takes the value $J(m)$ and the loop starts its execution again.

Figures 8 and 10 show curves obtained from the previous algorithm. The first curve represents the value of $J(m)$ for $m$ $=14 \mathrm{~m}^{2}$, the other one represents also the value of $J(\mathrm{~m})$ for a new value of $m=60 \mathrm{~m}^{2}$. Figures 9 and 11 show how points $\beta_{i j}$ converge to a single points that is, then, the solution of the geometric problem.

Although theoretically appealing, this process requires some interpolation and optimization process that have been handcrafted. Indeed, it is not easy how to incorporate a more involved algorithm not only to locate the vessel but to include a tracking perspective. Deep Neural Networks in next section will provide a very natural solution to these demands and shows very satisfactory performance.

\section{A. Location Using Deep Neural Networks}

1) Problem Description: Assume that we have the magnetic field measures $B\left(x_{t}, y_{t}, z_{t}, x_{s}, y_{s}, z_{s}, m_{1}, m_{2}\right)$, where $x, y$ and $z$ are the coordinates of the target (i.e., the ship) and the sensor, using subscripts $t$ and $s$ respectively. A question that arises is whether it is possible to locate the target by only having the field measured by a set of sensors, as well as the sensor coordinates.

This problem is known as state estimation [27]. In this problem, there is a state vector $\boldsymbol{x}$, which is unknown and needs to be estimated using a vector of observations $\boldsymbol{o}$ related to the state following a certain probability distribution function $P(\boldsymbol{x} \mid \boldsymbol{o})$. If $P(\boldsymbol{x} \mid \boldsymbol{o})$ is known, the state can be estimated using Bayesian filtering [28]. If the function $P(\boldsymbol{x} \mid \boldsymbol{o})$ is unknown, it is 


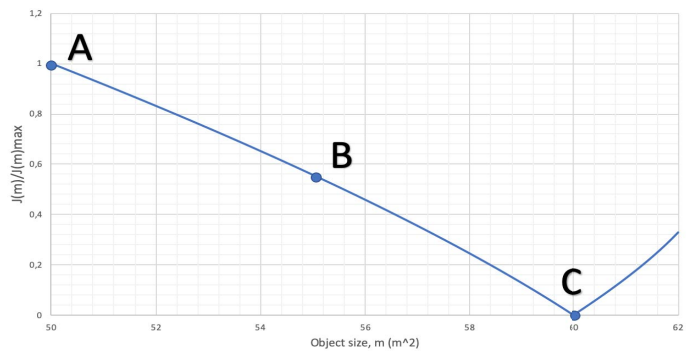

Fig. 10. Nomalizated results of $J(m)$ for an object size of $60 \mathrm{~m}^{2}$.

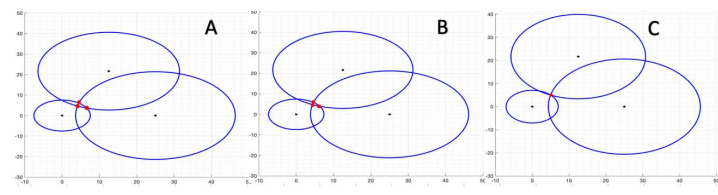

Fig. 11. Representation of the convergence o $\beta_{i j}$ solutions for an object size of $60 \mathrm{~m}^{2}$.

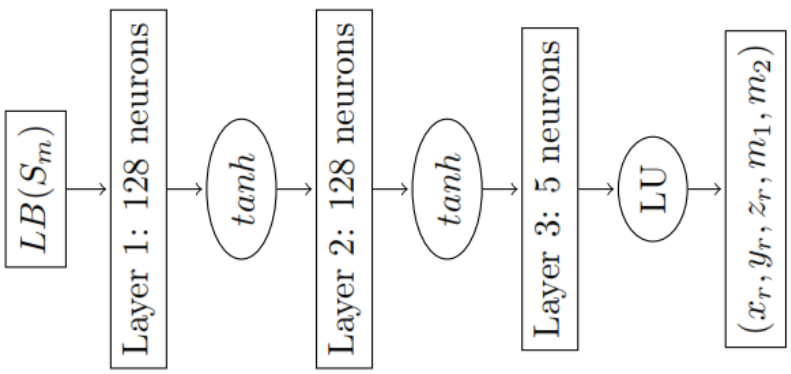

Fig. 12. DNN structure used to locate. We use a three layer feedforward network, where tanh denotes hyperbolic tangent activation functions and $L U$ stands for linear activation units.

possible to learn it by using an approach based on Deep Neural Networks (DNNs), such as [29] or [30]. DNNs are known to be universal function approximators [31] and hence, if they are chosen and trained adequately, they can approximate an arbitrary function.

In our case, we use a DNN to estimate the position of the target $\left(x_{t}, y_{t}, z_{t}\right)$ and its dimensions $\left(m_{1}, m_{2}\right)$. As input to our DNN, we use a transformation $L B\left(S_{m}\right)$ of the magnetic field measures $B\left(S_{m}\right)$ given by a subset of $m$ sensors $S_{m}$, where $L B\left(S_{m}\right)=\operatorname{sign}\left(B\left(S_{m}\right)\right) \log \left|B\left(S_{m}\right)\right|$. The transformation allows us to better capture the relation between distance and field, and also, preserve the sign information. Note that $L B\left(S_{m}\right)$ is a vector which contains $m$ magnetic field measurements. The DNN output vector is a 5-vector $\left(x_{r}, y_{r}, z_{r}, m_{1}, m_{2}\right)$, where the subscript $r$ indicates coordinates relatives to the first sensor of the $S_{m}$ set.

The structure of the DNN we use can be observed in Figure 12. Note that we make use of a simpler DNN structure than the ones used in [29] or [30]: instead of using Recurrent Neural Networks, we use a three feedforward layer DNN. Yet, our DNN shows to be enough for our purposes, as next section shows.

2) Simulation Results: We train our DNN using the $B\left(S_{m}\right)$ computer generated values. Namely, we have the $B\left(S_{m}\right)$ measures on a grid of $6328\left(x_{s}, y_{s}\right)$ points for $x_{s} \in[-100,100]$, $y_{s} \in[-50,50]$. We have a grid of measures for each of the 16 possible combinations of $z_{s}=\{7.5,10,20,30\}$ and
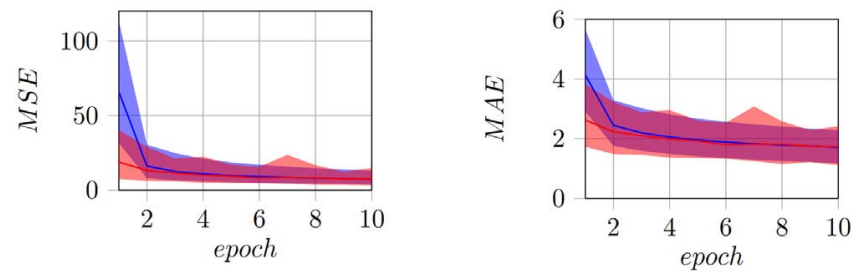

Fig. 13. Example of training results for the case $m=9$. We plot the mean values on the training set (blue) and validation set (red) and the shadowed region contains the maximum and minimum values obtained during the 30 different training processes. We observe how the MSE, the loss to minimize during training, decreases with the number of epochs. The same happens with the MAE. We note that there is no overfitting: MAE and MSE are very similar on both training and validation sets.

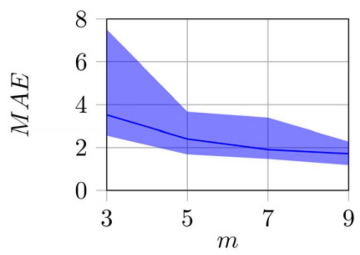

Fig. 14. MAE results as a function of $m$. We plot the mean final values of MAE on the training set. The shadowed region contains the maximum and minimum values obtained during the 30 different training processes. Note how the average value and the spread decrease as $m$ increases.

$\left(m_{1}, m_{2}\right)=\{(2,1),(4,2),(8,4),(20,10)\}$. Note that $x, y, z$ and $m$ units are meters.

In order to show that our DNN generalizes, we train it on different $S_{m}$ sets. Prior to each training procedure, we randomly choose $m$ sensors from the grid, with the only constrain that their distances are smaller than 15 meters, since the communication range of the sensors is 20 meters. Then, we train the network using 911232 training vectors and validate using 101248 different vectors, using 10 epochs over the whole training database and using Adam as optimizer [27] with parameters $\alpha=0.001, \beta_{1}=0.9, \beta_{2}=0.999$ and $\epsilon=10^{-8}$. The loss function we use is the mean squared error (MSE) and we also obtain the mean absolute error (MAE).

Each training procedure described above is repeated 30 times, in order to use 30 different $S_{m}$ vectors. We train using $m=\{3,5,7,9\}$ sensors, in order to observe how the number of sensors affect the DNN results. The results can be observed in Figures 13 and 14. In Figure 14 we plot the MSE and MAE evolution during the training process for the case $m=9$. Note how there is improvement with the number of epochs and how there is no overfitting, since the validation MSE and MAE are very similar to the training ones. Similar results (with different error values) are obtained for the rest of $m$ values.

Note that each time the DNN is trained, the sensor set $S_{m}$ changes. This means that the $\theta$ parameters of the DNN are trained specifically for each concrete sensor set. In other words, we train the DNN knowing the number and position of the sensors. In a setup where the sensor positions are not known before being deployed, this means that we require the sensors to be located after deployment and then, training the DNN.

In Figure 14, we plot the final MAE values obtained in the training set as a function of $m$. Note how the mean and extreme values of MAE decrease as $m$ increases. This was to 

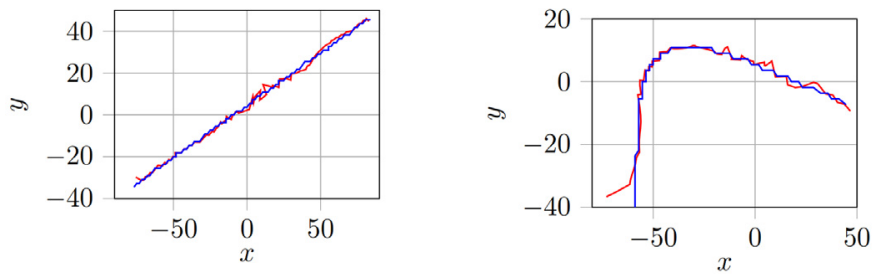

Fig. 15. Example of trajectories predicted using the trained DNN when $m=9, z_{t}=7.5, m_{1}=2, m_{2}=1$. Blue line is the actual target trajectory, and the red line is the trajectory predicted by the DNN. The MAE for the $\left(x_{t}, y_{t}\right)$ values is 1.19 meters for the first trajectory and 0.99 meters for the second.

be expected: the more information we have, the most accurate our prediction will be. Also, observe how the MAE is around 2 meters for $m=\{7,9\}$. This means that the mean absolute error between the predicted position of the target and its dimensions, and the actual position and dimensions, is in the order of a couple of meters per dimension. This result means that the prediction given by our DNN is quite good.

In order to observe better what the DNN predicts, we plot the prediction results for two different example trajectories in Figure 15. There, we observe that the predictions given by the DNN proposed are quite good, predicting with a low absolute error the position of the target.

Finally, we remark that a higher accuracy could be obtained in the prediction of the trajectory by using tools for trajectory estimation that take into account information about the speed and acceleration $(\alpha-\beta-\gamma$ filter, see [27]), information about the probability distribution function of the trajectory (Kalman filter, see [27]), or even using Neural Networks to learn this (as in [29]). Thus, the results obtained could be further improved in concrete scenarios where more information about the trajectory is known.

\section{CONCLUSION}

In this work we have proposed a new system for the identification, surveillance and monitoring of boats of medium size in areas of shallow water. The main objective is to estimate the size of the object and the followed trajectory as accurate as possible. To achieve this, a network of underwater nodes has been designed with a series of magnetic sensors.

In this work, a description is made of the electronics that handle the magnetic sensors that are fluxgate type. Our final set-up that it is quite simple and cheap, which allows a large number of nodes to be put into operation, which makes it possible to create a network with much better resolution and much more extensive.

To justify the use of magnetic sensors, a series of simulations have been carried out using software specialized in magnetic simulations. The AMPERES program has provided important information about the detected field values for various object sizes and several distances. The processing of the obtained data has served to design an algorithm based on triangulation of nodes to perform for the identification and monitoring of the vessels.

However, this algorithm offers information that is not very accurate and in some circumstances, showing some ambiguities. Therefore we present the optimal solution for the problem based on a neural network with full capability to approximate the modeled mapping between the magnetic model and the classification and estimator results. Its application, on the processed data of the simulations allows to know with higher accuracy both the size of the object and its trajectory, with a margin of error very small.

In the future we will try to corroborate these results with real measurements.

\section{REFERENCES}

[1] Underworld Project. Accessed: 2016. [Online]. Available: http://www. underworldproject.eu

[2] E. Cayirci, H. Tezcan, Y. Dogan, and V. Coskun, "Wireless sensor networks for underwater surveillance systems," Ad Hoc Netw., vol. 4, pp. 431-446, Jul. 2006.

[3] I. F. Akyildiz, D. Pompili, and T. Melodia, "Underwater acoustic sensor networks," Ad Hoc Netw., vol. 3, pp. 257-279, May 2005.

[4] A. Bahr, J. J. Leonard, and M. F. Fallon, "Cooperative localization for autonomous underwater vehicles," Int. J. Robot. Res., vol. 28, no. 6 , pp. 714-728, 2009.

[5] S. E. Webster, R. M. Eustice, H. Singh, and L. L. Whitcomb, "Preliminary deep water results in single-beacon one-way-travel-time acoustic navigation for underwater vehicles," in Proc. IEEE/RSJ Int. Conf. Intell. Robots Syst., Oct. 2009, pp. 2053-2060.

[6] X. Cheng, H. S. Haining Shu, and Q. Liang, "A range-difference based self-positioning scheme for underwater acoustic sensor networks," in Proc. Int. Conf. Wireless Algorithms, Syst. Appl. (WASA), Aug. 2007, pp. $38-43$.

[7] D. Frye et al., "An acoustically-linked deep-ocean observatory," in Proc. Eur. Oceans, Jun. 2005, pp. 969-974.

[8] N. Farr, A. Bowen, J. Ware, C. Pontbriand, and M. Tivey, "An integrated, underwater optical /acoustic communications system," in Proc. Oceans IEEE Sydney, May 2010.

[9] E. Demirors, G. Sklivanitis, T. Melodia, S. N. Batalama, and D. A. Pados, "Software-defined underwater acoustic networks: Toward a high-rate real-time reconfigurable modem," IEEE Commun. Mag., vol. 53, no 11, pp. 64-71, Nov. 2015.

[10] U. Datta, R. Otnes, and C. Lucas, "Bearing estimation using small tetrahedral passive hydrophone array," in Proc. Oceans MTS/IEEE Seattle, Sep. 2010, pp. 1-8, doi: 10.1109/OCEANS.2010.5664345.

[11] M. Erol-Kantarci, H. T. Mouftah, and S. Oktug, "A survey of architectures and localization techniques for underwater acoustic sensor networks," IEEE Commun. Surveys Tuts., vol. 13, no. 3, pp. 487-502, 3rd Quart., 2011.

[12] T. Melodia, H. Kulhandjian, L.-C. Kuo, and E. Demirors, "Advances in underwater acoustic networking," in Mobile Ad Hoc Networking: Cutting Edge Directions, S. Basagni, M. Conti, S. Giordano, I. Stojmenovic, Eds., 2nd ed. Hoboken, NJ, USA: Wiley, 2013, pp. 804-852.

[13] E. Felemban, F. K. Shaikh, U. M. Qureshi, A. A. Sheikh, and S. B. Qaisar, "Underwater sensor network applications: A comprehensive survey," Int. J. Distrib. Sensor Netw., vol. 11, no. 11, Nov. 2015, Art. no. 896832.

[14] H.-P. Tan, R. Diamant, W. K. G. Seah, and M. Waldmeyer, "A survey of techniques and challenges in underwater localization," Ocean Eng., vol. 38, nos. 14-15, pp. 1663-1676, Oct. 2011.

[15] G. Han, J. Jiang, L. Shu, Y. Xu, and F. Wang, "Localization algorithms of underwater wireless sensor networks: A survey," Sensors, vol. 12, pp. 2026-2061, Feb. 2012.

[16] V. Chandrasekhar, W. K. Seah, Y. S. Choo, and H. V. Ee, "Localization in underwater sensor networks: Survey and challenges," in Proc. 1st ACM Int. workshop Underwater Netw. - WUWNet, 2006, pp. 33-40.

[17] Z. Zhou, Z. Peng, J.-H. Cui, Z. Shi, and A. Bagtzoglou, "Scalable localization with mobility prediction for underwater sensor networks," IEEE Trans. Mobile Comput., vol. 10, no. 3, pp. 335-348, Mar. 2011.

[18] J. Zazo et al., "Underwater electromagnetic sensor networks-Part II: Localization and network simulations," Sensors, vol. 16, no. 12, p. 2176, Dec. 2016.

[19] H. Niu, E. Reeves, and P. Gerstoft, "Source localization in an ocean waveguide using supervised machine learning," J. Acoust. Soc. Amer, vol. 142, no. 3, pp. 1176-1188, Sep. 2017.

[20] Z. Huang, J. Xu, Z. Gong, H. Wang, and Y. Yan, "A deep neural network based method of source localization in a shallow water environment," in Proc. IEEE Int. Conf. Acoust., Speech Signal Process. (ICASSP), Apr. 2018, pp. 3499-3503. 
[21] J. Parras, S. Zazo, I. A. Pérez-Álvarez, and J. L. S. González, "Model free localization with deep neural architectures by means of an underwater WSN," Sensors, vol. 19, no. 16, p. 3530, Jan. 2019.

[22] J. A. Catipovic, "Performance limitations in underwater acoustic telemetry," IEEE J. Ocean. Eng., vol. 15, no. 3, pp. 205-216, Jul. 1990.

[23] B. Tamayo et al., "Magnetometric sensor to control the ground traffic of aircraft," Sens. Actuators A, Phys., vols. 37-38, pp. 442-448, Jun. 1993.

[24] Integrated Engineering Software, Amperes, Santa Clara, CA, USA, 2012.

[25] M. Díaz-Michelena, "Small magnetic sensors for space applications," Sensors, vol. 9, no. 4, pp. 2271-2288, Mar. 2009.

[26] G. Quintana-Díaz et al., "Underwater electromagnetic sensor networks, Part I: Link characterization," Sensors, vol. 17, no. 1, p. 189, 2017.

[27] D. Simon, Optimal State Estimation: Kalman, H Infinity, and Nonlinear Approaches. Hoboken, NJ, USA: Wiley, 2006.

[28] M. S. Arulampalam, S. Maskell, N. Gordon, and T. Clapp, "A tutorial on particle filters for online nonlinear/non-Gaussian Bayesian tracking," IEEE Trans. Signal Process., vol. 50, no. 2, pp. 174-188, Aug. 2002.

[29] P. Ondrúška and I. Posner, "Deep tracking: Seeing beyond seeing using recurrent neural networks," in Proc. 13th AAAI Conf. Artif. Intell., 2016, pp. 3361-3367.

[30] A. Milan, S. H. Rezatofighi, A. R. Dick, I. D. Reid, and K. Schindler, "Online multi-target tracking using recurrent neural networks," in Proc. 31st AAAI Conf. Artif. Intell., 2017, pp. 4225-4232.

[31] K. Hornik, "Approximation capabilities of multilayer feedforward networks," Neural Netw., vol. 4, no. 2, pp. 251-257, 1991.

[32] D. P. Kingma and J. Ba, "Adam: A method for stochastic optimization," 2014, arXiv:1412.6980. [Online]. Available: http://arxiv.org/ abs/1412.6980

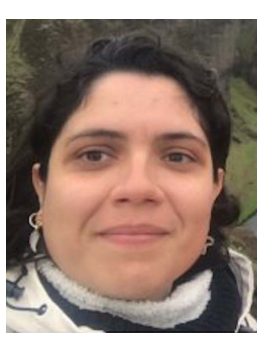

Marina Pérez received the B.S. and M.S. degrees in telecommunication engineering from the Technical University of Madrid in 2011 and 2014, respectively. He is currently pursuing the Ph.D. degree in physical electronics with the Telecommunication Engineering School, UPM. His research interests include magnetic sensors, microprocessors, underwater communications, aerospace technology, and communications based on magnetic induction.

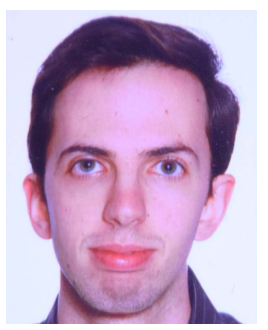

Juan Parras received the B.S. degree in telecommunications engineering from Universidad de Jaén in 2014 and the M.Sc. degree in telecommunications engineering from Universidad Politécnica de Madrid (UPM) in 2016, where he is currently pursuing the Ph.D. degree in telecommunications engineering. His research interests include audio and radio signal processing and game theory applied to communications networks.

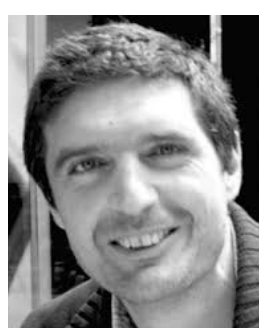

Santiago Zazo (Member, IEEE) is currently a Telecom Engineer and Dr. Engineer with Universidad Politecnica de Madrid (UPM), Madrid, Spain, since 1990 and 1995, respectively. From 1991 to 1994, he was with the University of Valladolid, Valladolid, Spain, and with the University Alfonso X El Sabio, Madrid, Spain, from 1995 to 1997. In 1998, he joined UPM as an Associate Professor in signal theory and communications. His main research activities are in the field of signal processing, initially with applications to audio, communications and radar. More recently, he has been focused on MIMO communications and wireless sensor networks from both physical layer and networking points of view. Since 1990, he has coauthored more than 40 journal articles and about 200 conference papers. Since 2016, he has been a Full Professor with UPM. His main activity at this moment is related to distributed algorithms, optimization, and game theory with special focus on dynamic games with perfect and imperfect information.

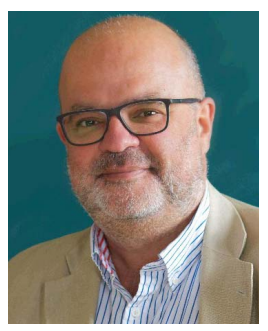

Iván A. Pérez Álvarez (Member, IEEE) received the Telecom Engineering and Dr. Engineering degrees from the Universidad Politécnica de Madrid (UPM), Madrid, Spain, in 1990 and 2000, respectively. From 1989 to 1997, he worked for Europea de Comunicaciones S.A. and Telefónica Sistemas S.A., where he was a staff member of the Special Projects Department and worked with the Digital Signal Processing Group. He was involved in the design of HF/VHF/UHF digital communications systems for the Spanish Ministry of Defense. He joined the Universidad de Las Palmas de Gran Canaria (ULPGC), as an Associate Professor, in 1998. From 2006 to 2010, he was the Head of the Centre for the Technological Centre for Innovation in Communications (CeTIC), and from 2010 to 2016, he was a Vice-Director of the Institute for Technological Development and Innovation in Communications (IDeTIC). He is currently the Head of the Telecommunications and Electronic Engineering School. His research interest includes signal processing for radio communications.

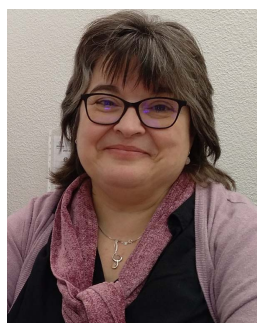

María del Mar Sanz Lluch received the Ph.D. degree in physics from Universidad Complutense de Madrid in 1995. She joined Universidad Politécnica de Madrid in 2005 as an Associate Professor. Her research areas include diagnostic and modeling of cold plasmas and magnetic nanoparticles synthesis. She is currently working in the simulation and development of magnetic devices and their characterization. 